\title{
Efectos de la dexmedetomidina sobre la hemodinámica intracraneal en pacientes con lesión encefálica traumatica grave
}

\author{
P. Grille; A. Biestro; G. Fariña y R. Miraballes \\ Unidad de Cuidados Intensivo. Hospital de Clínicas. Universidad de la República. Montevideo. Uruguay.
}

\section{Resumen}

La dexmedetomidina, un agonista adrenérgico selectivo $\alpha 2$, constituye un intento de mejorar la sedoanalgesia de los pacientes críticos, por sus efectos distintivos en comparación a los planes tradicionales como son: sedación consciente, simpaticolisis y ausencia de depresión respiratoria.

Objetivo. Estudiar el efecto de la dexmedetomidina, un nuevo agonista $\alpha 28$, sobre la hemodinámica intracraneal y sobre los parámetros hemometabólicos cerebrales en un grupo de pacientes con trauma craneoencefálico grave.

Material y métodos. Estudio prospectivo de los pacientes con lesión encefálica traumática grave (Glasgow Coma Scale $\leq 8$ ) ingresados en un Centro de Tratamiento Intensivo (CTI) que recibieron monitorización de la presión intracraneal (PIC) y monitorización de saturación de $\mathrm{O}_{2}$ del bulbo yugular $\left(\mathrm{SjO}_{2}\right)$. Se realizó una perfusión intravenosa de la droga durante 3 horas, en dosis progresivas $(0.2,0.4$ y $0.7 \mathrm{ug} / \mathrm{kg} / \mathrm{h})$, previa suspensión de otros sedantes y analgésicos.

Resultados. Se estudiaron 12 pacientes sin hipertensión intracraneal. No se encontraron diferencias significativas entre los valores de PIC y presión arterial media (PAM) tras la infusión de la droga en relación con los valores basales. La presión de perfusión cerebral (PPC) mostró una tendencia a disminuir durante el estudio (efecto marginal, $p=\mathbf{0 . 0 5 8})$. Se encontró un descenso significativo de la frecuencia cardíaca $(F C)(p<0.0001)$ en relación a los valores basales. No se hallaron diferencias significativas en los parámetros hemometabólicos cerebrales $\left(\mathrm{SjO}_{2}\right.$ y $\left.\mathrm{CEO}_{2}\right)$.

Conclusión. A las dosis utilizadas, la dexmedetomidina fue segura, no asociándose a alteraciones significativas de la hemodinámica intracraneal ni del metabolismo sanguíneo cerebral en pacientes en la etapa aguda del trauma craneoencefálico grave (TECG)

PALABRAS CLAVE: Dexmedetomidina. Lesión encefálica traumática grave. Trauma craneal. Presión intracraneal.

Recibido: 3-09-04. Aceptado: 15-11-04
Presión de perfusión cerebral. Hemodinámica intracraneal. Saturación de oxígeno yugular

Effects of dexmedetomidine on intracranial hemodynamics in severe head injured patients

\section{Summary}

Dexmedetomidine, an $\alpha 2$ adrenergic agonist, with distinctive characteristics when compared to traditional plans, namely: conscious sedation, sympatholysis and lack of respiratory depression, represents an attempt to improve the sedoanalgesia of critically ill patients.

Objective. To study dexmedetomidine's effect on intracranial hemodynamic and hemometabolic parameters in severe head injured patients.

Material and methods. Prospective study on the effect of Dexmedetomidine on twelve severe head injured patients (Glasgow Coma Scale score $\leq 8$ ) admitted to an intensive care unit, with intracranial pressure < $20 \mathrm{mmHg}$ and $\mathrm{O}_{2}$ saturation monitoring of blood from jugular bulb. The drug was perfused intravenously during 3 hours, in progressive doses $\left(\begin{array}{lllll}0.2 & 0.4 & \text { y } & 0.7\end{array}\right.$ $\mathrm{ug} / \mathrm{kg} / \mathrm{h})$. All other sedo-analgesia medication had been previously withdrawn.

Results. No significant differences were found in intracranial pressure, mean arterial pressure and cerebral hemometabolic parameters after infusion of dexmedetomidine in relation to basal values. Cerebral perfusion pressure showed a trend to decrease during the drug infusion (marginal effect, $p=.058$ ). Cardiac frequency decreased significantly after the drug admi-

Abreviaturas. APACHE: acute physiological and chronic health evaluation. $\mathrm{CEO}_{2}$ : coeficiente extracción de oxígeno. $\mathrm{paCO}_{2}$ : presión deCO ${ }_{2}$. CTI: centro de tratamiento intensivo. DE: desviación estándar. FC: frecuencia cardiaca. FSC: flujo sanguineo cerebral. GCS: Glasgow Coma Scale. HIC: hipertensión intracraneal. PA: presión arterial. PAM: presión arterial media. PIC: presión intracraneal. PPC: presión de perfusión cerebral. $\mathrm{SjO}_{2}$ : saturación de oxígeno bulbo yugular. TCEG: traumatismo craneoencefálico grave. VSC: volumen sanguíneo cerebral. 


\section{nistration.}

Conclusions. At the doses utilized, dexmedetomidine was safe, and it was not associated with significant changes in intracranial hemodynamics, nor in cerebral hemometabolic parameters, in a group of severe head jnjured patients.

KEY WORDS: Dexmedetomidine. Head injury. Head trauma. Intracranial pressure. Cerebral perfusion pressure. Intracranial hemodynamics. Oxygen jugular saturation.

\section{Introducción}

La sedo-analgesia es considerada un componente esencial de la práctica clínica en el manejo terapéutico de los pacientes críticos y especialmente en los pacientes con lesión neurológica grave ${ }^{17,24,25,29}$. Sin embargo, no hay estudios que demuestren niveles de evidencia altos respecto a su influencia en el resultado final de estos enfermos. A pesar de ello, el tema es muy importante pues: a) resulta una medida terapéutica constante en esta población de pacientes, b) los fármacos utilizados pueden tener múltiples efectos y algunos de ellos -no bien reconocidospueden operar sobre la hemodinámica intracraneana, c) no existe un régimen único y ya ha habido algún reporte que ha mostrado diferencias significativas sobre la presión intracraneal (PIC) de diferentes planes de sedo-analgesia ${ }^{15}$, y d) representa, por su uso muy frecuente y prolongado, una cuota no despreciable de los recursos económicos gastados en estos pacientes.

El criterio actual de sedo-analgesia en pacientes neurolesionados ha cambiado en los últimos años ${ }^{14}$. Aunque no existe un régimen ideal, que haya sido universalmente aceptado para este tipo de pacientes, hay preferencia por regímenes que ofrezcan tranquilidad con eventual control de la postura tónica, analgesia, cierta relajación y que, por sus características farmacodinámicas, permitan la evaluación clínica neurológica a corto tiempo de suspendida la infusión ("ventana de sedo-analgesia"). En la fase de despertar, estos pacientes pueden sufrir, también, estados de agitación, por lo que en estos casos se prefieren los fármacos que sin deprimir el estado de conciencia controlen la excitación sicomotriz (sedación consciente).

El nuevo agente de la clase de los agonistas de los adrenorreceptores $\alpha 2$, dexmedetomidina, podría tener un lugar en el tratamiento de los pacientes con lesión encefálica grave en el manejo de los escenarios clínicos previamente señalados ${ }^{1,2,3,5,12,13,26}$. La dexmedetomidina brinda tranquilidad efectiva con propiedades analgésicas, lo cual permite abolir $\mathrm{y} / \mathrm{o}$ reducir la necesidad de otros sedantes y analgésicos, sin provocar depresión respiratoria. Presenta, además, efectos benéficos para este tipo de pacientes tales como: ansiolisis, simpaticolisis, así como su escaso efecto amnésico, lo cual puede tener ventajas en la etapa de despertar $^{3,5}$. Además, se ha demostrado en animales cierto papel neuroprotector, cuyo mecanismo no se conoce bien, proponiéndose se deba a la disminución de la liberación de noradrenalina sistémica y/o intracerebral ${ }^{7,8,11,17,19}$. Debido a su mecanismo de acción selectivo $\alpha 2$, se asocia con efectos hemodinámicos predecibles, lo que disminuye sus perjuicios potenciales ${ }^{1,4,28}$. Hay pocos datos sobre la acción que la dexmedetomidina pueda tener sobre la hemodinámica intracraneana en seres humanos. Además, hasta lo que nosotros conocemos, no hay datos de su acción en el grupo de pacientes con TECG. Estos pacientes pueden tener disminución de la "compliance" intracraneana por lo que los efectos de la droga sobre la hemodinámica intracraneal deberían ser rigurosamente escrutados antes de su recomendación general.

El objetivo de este trabajo fue determinar el efecto de la dexmedetomidina sobre la PIC, la PPC, así como también su comportamiento en relación a los parámetros hemometabólicos cerebrales y temperatura sistémica en pacientes con TECG.

\section{Material y métodos}

Se realizó un estudio prospectivo de los pacientes ingresados al CTI del Hospital de Clínicas, Montevideo, Facultad de Medicina, Universidad de la República, y que cumplían con los siguientes criterios de inclusión: pacientes con TECG (Glasgow Coma Scale $\leq 8$ ) cerrado y monitorización de la PIC y de la presión arterial (PA) en forma invasiva; edad comprendida entre 15 y 65 años. Se excluyeron del estudio: a) pacientes que presentaban al inicio del estudio hipertensión intracraneal (HIC); b) pacientes que presentaban inestabilidad hemodinámica, entendida ésta, como la necesidad de reposición de volumen o ajuste de dosis de inotrópicos frecuente para mantener una presión arterial y perfusión sistémica estable; c) pacientes con bloqueo de conducción avanzada (bloqueo aurículo-ventricular completo o de segundo grado, trifasicular); d) paciente embarazada; e) pacientes con insuficiencia renal o hepática graves.

Se obtuvo previamente la autorización mediante consentimiento informado del enfermo o responsable de cada paciente, acerca del motivo y características del estudio.

La monitorización de los pacientes incluyó: PA invasiva mediante catéter en arteria radial (Datex, Solo); PIC mediante: sistema parenquimatoso (Codman MicroSensors ICP Transducer, Raynham, MA) en 6 pacientes, catéter subdural hidráulico en 5 pacientes y catéter ventricular en el restante; oximetría de pulso; y análisis de la saturación yugular de oxígeno $\left(\mathrm{SjO}_{2}\right)$ en forma discontinua mediante muestras analizadas con co-oxímetro (Radiometer, ABL 530). En algunos pacientes se efectuó monitorización de 
la temperatura rectal y timpánica durante el tiempo que se efectuó la perfusión de la droga mediante termopar (MonA-Therm, Mallinckrodt).

El manejo de los pacientes se basó en las Guías consensuadas de Manejo de la lesión Encefálica Traumática Grave recientemente publicadas ${ }^{24}$.

El protocolo de estudio consistió en la perfusión intravenosa, por bomba de infusión, de dexmedetomidina (Precedex, Laboratorios Abbott) durante un período de 3 horas. La droga en estudio se preparó mediante el agregado de 1 ampolla de $2 \mathrm{ml}$ de clorhidrato de dexmedetomidina (200 ug) a $48 \mathrm{ml}$ de Cloruro de Sodio al 0.9\% (concentración final: $4 \mathrm{ug} / \mathrm{ml})$. Se utilizaron dosis crecientes, recomendadas por el laboratorio fabricante : $0.2 \mathrm{ug} / \mathrm{kg} / \mathrm{h}$ la primera hora, $0.4 \mathrm{ug} / \mathrm{kg} / \mathrm{h}$ la segunda hora, y $0.7 \mathrm{ug} / \mathrm{kg} / \mathrm{h}$ la tercera hora. Se realizaron medidas de PIC, PPC, así como de saturación yugular de oxígeno $\left(\mathrm{SjO}_{2}\right)$ e índice de extracción cerebral de oxígeno $\left(\mathrm{CEO}_{2}\right)$ estimado como la diferencia entre la saturación arterial y la $\mathrm{SjO}_{2}$ (como estimaciones globales del metabolismo cerebral de oxígeno). Todos estos parámetros fueron medidos cada 15 minutos.

Durante el período de estudio la $\mathrm{paCO}_{2}$ se mantuvo constante, con una media de $36,5 \pm 0,95 \mathrm{mmHg}$.

Si el paciente estaba recibiendo otra sedación, previamente al inicio del estudio, se suspendió la misma, por lo menos 2 horas antes del inicio del estudio.

Como información general de los pacientes se registraron: edad, sexo, días de estancia, puntuación del GCS, puntuación fisiológica APACHE II al ingreso al $\mathrm{CTI}^{16}$, clasificación tomográfica de $\mathrm{Marshall}^{20}$, y necesidad de neurocirugía.

Todos los datos demográficos se expresaron como la media \pm desvío estándar (DE). Para analizar el efecto de la dexmedetomidina, se realizó el análisis de varianza (ANOVA) de medidas repetidas, no paramétricas (Test de Friedman), considerándose significativo un valor de $\mathrm{p}<$ 0.05 .

\section{Resultados}

Se estudiaron 12 pacientes. Las características demográficas y clínicas de los mismos se muestran en la Tabla I.

Los valores basales de los parámetros estudiados y sus cambios con la perfusión progresiva de dexmedetomidina se muestran en la tabla II.

Los valores de PIC y PAM no variaron significativamente durante el estudio, en relación con los valores basales. Los valores de PPC mostraron una tendencia a disminuir (efecto marginal, con un valor de $\mathrm{p}=0.058$ con respecto a los valores basales), siendo el punto de mayor significancia a los 150 min de la infusión (dosis: $0.07 \mathrm{ug} / \mathrm{kg} / \mathrm{h}$ ) según el análisis post-test $(83+/-11 \mathrm{mmHg}$ versus $92+/-19 \mathrm{mmHg})$ (Tabla II y Figuras 1 y 2).
Tabla I

Caracteristicas clínicas de los pacientes estudiados

\begin{tabular}{lcc} 
Características & & \\
\hline Número de pacientes (n) & 12 & \\
Edad (años) & $33 \pm 15$ & \\
Sexo (F / M) & $1 / 9$ & \\
Clasificación TC de Marshall & I & 1 \\
& II & 5 \\
& III & 2 \\
GCS inicial (media) & V & 4 \\
APACHE II & $6 \pm 2$ & \\
Neurocirugía & $20 \pm 6$ & \\
Estadía en CTI (días) & $5 / 12$ & \\
Mortalidad en CTI & $31 \pm 22$ & \\
Día de realización de prueba (días) & $1 / 12(10 \%)$ \\
\end{tabular}

No fue necesario la reposición de volumen ni el agregado y/o aumento significativo de las dosis de vasopresores para mantener la PAM por encima de $80 \mathrm{mmHg}$ durante el estudio.

Se halló un descenso significativo de la FC en relación a los valores basales ( $\mathrm{p}=0.0004)$, con la mayor significancia a los 150 min (93 vs $77 \mathrm{cpm}$ ) (Tabla II y Figura 3).

Los valores de $\mathrm{SjO}_{2}$ y $\mathrm{CEO}_{2}$ no variaron significativamente durante el estudio ( Tabla II y Figura 4).

En los pacientes estudiados no se comprobaron cambios significativos de la temperatura rectal y timpánica. (Tabla II y Figura 5)

En ningún caso el estudio debió ser detenido por efecto adverso de la droga.

\section{Discusión}

Los resultados auspiciados obtenidos con el uso de dexmedetomidina y otros $\alpha 2$ agonistas en la sedo-analgesia del peri-operatorio de cirugía cardíaca y vascular y sus propiedades específicas comparadas con los sedantes de uso habitual hacen prever un uso más difundido de la droga $a^{1,28,30}$. Antes de una recomendación general en el grupo de pacientes neurolesionados, deben estudiarse sus efectos sobre la hemodinámica intracraneal en el campo clínico, ya que no son previsibles, a priori, dada la acción compleja de la droga.

Por lo menos, 4 acciones de la droga deben ser tomadas en cuenta en el sentido de que pueden influenciar el resultado final sobre la hemodinámica intracraneal: 1) al actuar sobre los receptores $\alpha_{-2 \mathrm{~A}}$ presinápticos disminuye la liberación de noradrenalina, con lo que promueve la vasodilatación indirecta al disminuir la producción de 
Neurocirugía

Grille y col 2005; 16: 411-418

\begin{tabular}{|c|c|c|c|c|c|c|c|c|c|c|c|c|c|c|c|}
\hline 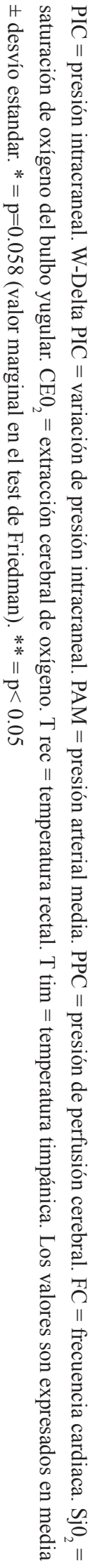 & $\begin{array}{l}\text { Oे } \\
\stackrel{\#}{0} \\
\stackrel{N}{\mathbb{H}}\end{array}$ & $\begin{array}{l}w \\
\dot{y} \\
\dot{0} \\
0 \\
\infty\end{array}$ & $\begin{array}{l}w \\
w \\
\infty \\
+ \\
\infty \\
\infty\end{array}$ & $\begin{array}{l}w \\
\infty \\
\dot{0} \\
0 \\
0 \\
0\end{array}$ & 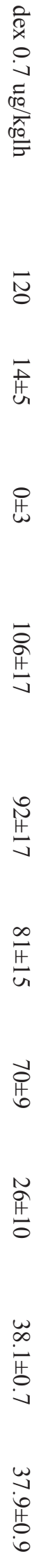 & $\begin{array}{l}\vec{O} \\
\dot{0} \\
\overrightarrow{+} \\
\overrightarrow{+}\end{array}$ & $\begin{array}{l}\omega \\
\infty \\
\dot{H} \\
\dot{0} \\
0\end{array}$ & 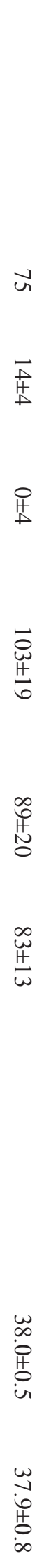 & 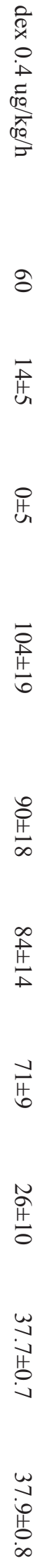 & 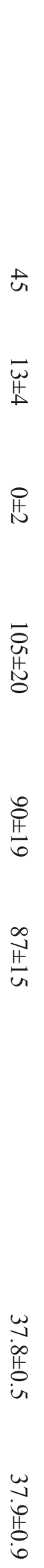 & $\begin{array}{l}w \\
\vec{y} \\
\infty \\
0 \\
\dot{0} \\
0\end{array}$ & $\begin{array}{l}w \\
\infty \\
\dot{0} \\
\dot{1} \\
\dot{0}\end{array}$ & 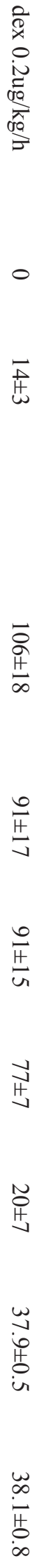 & 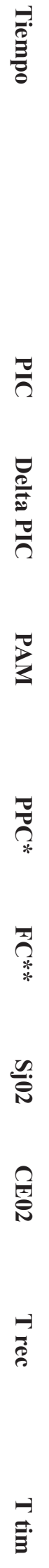 & 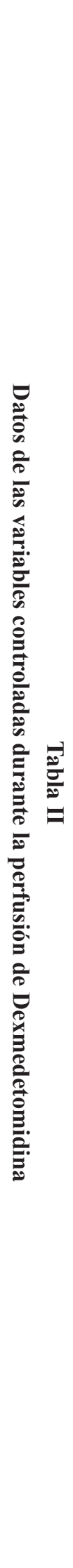 \\
\hline
\end{tabular}



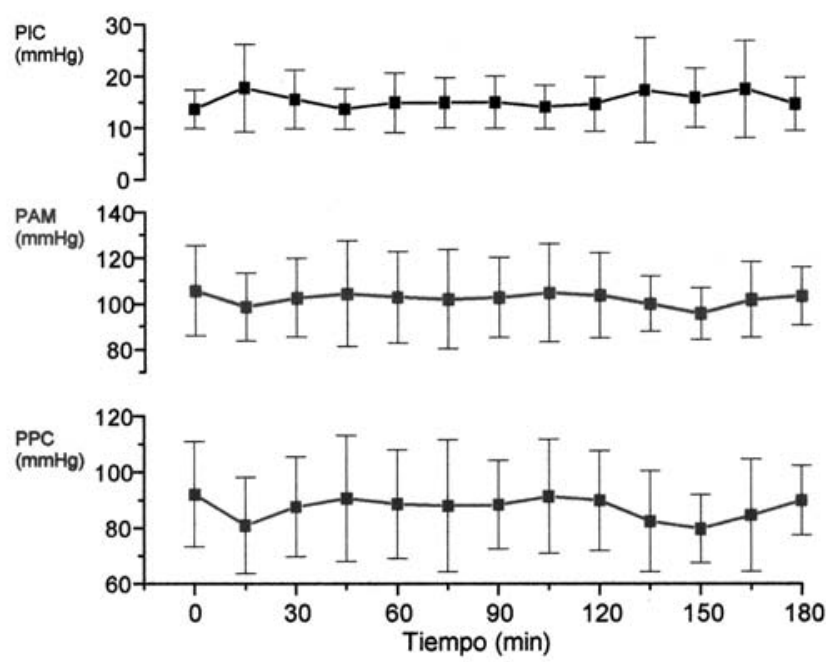

Figura 1. Valores de media de PIC, PAM y PPC durante la infusión de dexmedetomidina. $(p=N S)$

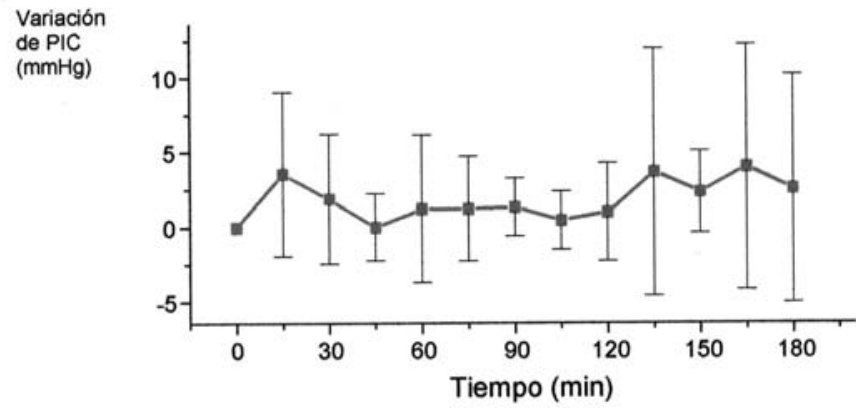

Figura 2. Valores de media de la variación de la PIC durante la infusión con dexmedetomidina $(p=N S)$

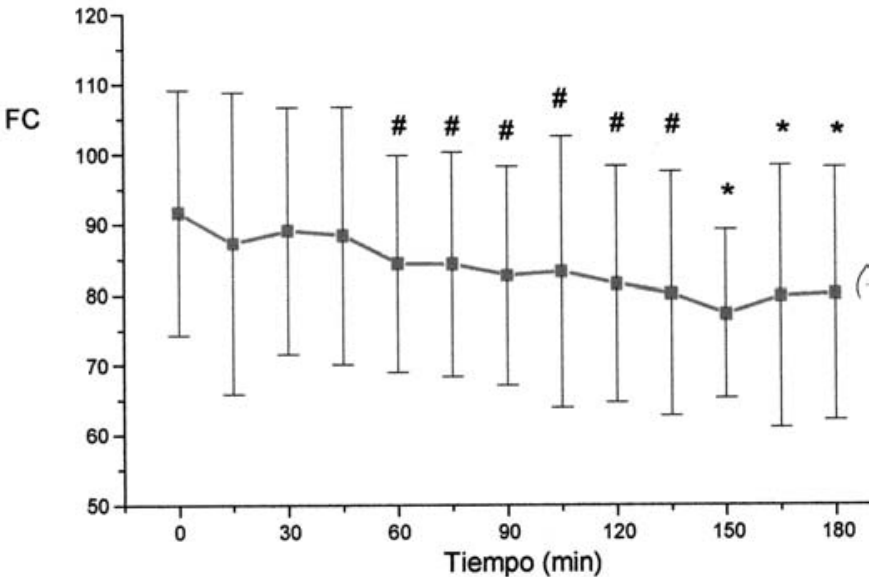

Figura 3. Valores de media de FC durante la infusión de dexmedetomidina. Diferencia significativa con los valores basales: $\# p<0.05 ; * p<0.001$

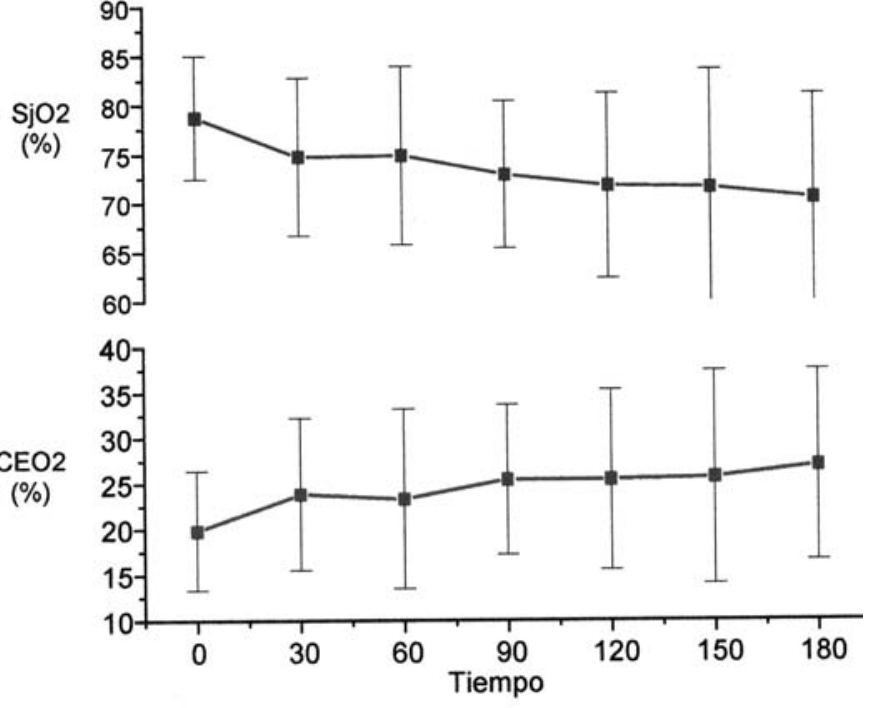

Figura 4. Valores de media de $\mathrm{SjO}_{2}$ e $\mathrm{CEO}_{2}$ durante la infusión de dexmedetomidina $(p=N S)$

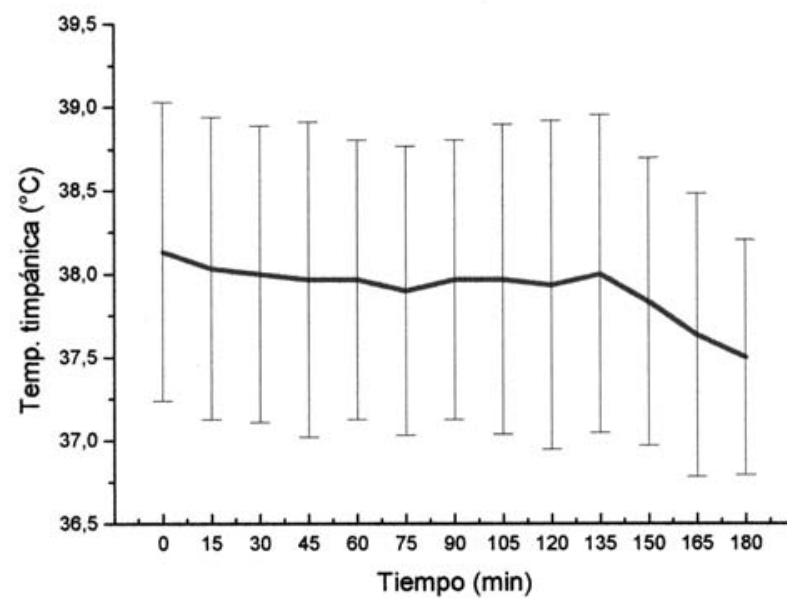

Figura 5. Valores de la media de T timpánica y rectal durante la infusión de dexmedetomidina $(p=N S)$

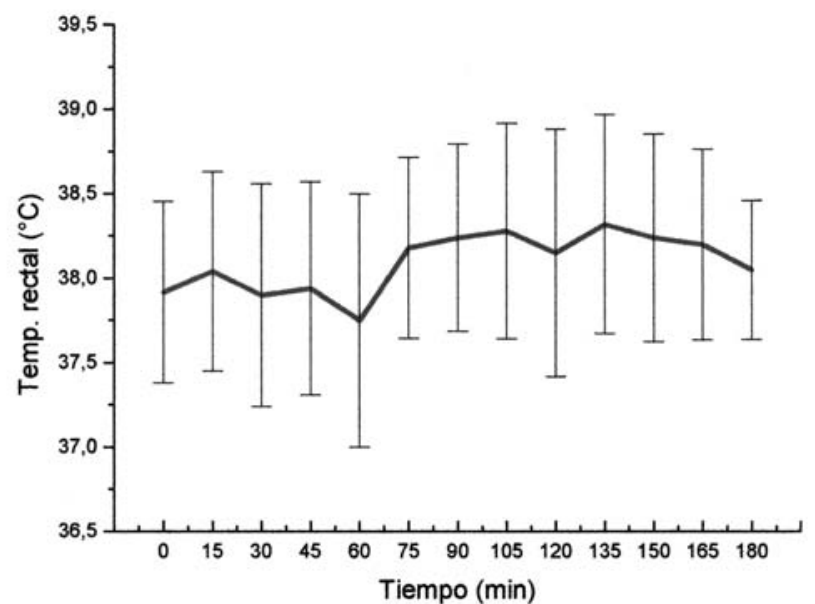

Figura 6. Valores de la media de la temperatura rectal durante la infusión de dexmedetomidina $(p=N S)$ 
endotelina -1 , y facilita la producción de oxido nítrico ${ }^{9,10}$, 2) al actuar sobre los receptores $\alpha_{-2 B}$ del músculo liso arteriolar puede incrementar directamente el tono y generar un efecto vasoconstrictor e hipertensor (regional del SNC pero también sistémico), lo cual logra, al parecer, a través de los canales tipo L de calcio y con dosis alta de la droga, 3) la sedación y tranquilidad generada por la droga, actuando sobre el "locus ceruleo" y mediante la estimulación del acople metabólico-vascular promovería vasoconstricción y consiguiente reducción del FSC por lo menos en algunas regiones del encéfalo, y por último 4) los efectos sistémicos de la droga, induciendo bradicardia e hipotensión, deberían activar los mecanismos de autorregulación con conservación del FSC pero con vasodilatación y consiguiente aumento del volumen sanguíneo cerebral (VSC).

Es difícil prever el efecto resultante que tendrá la interacción de estas fuerzas con distinto signo sobre el FSC y el VSC. En humanos voluntarios sanos estudiados con PET, la droga mostró un efecto de disminución del FSC global ${ }^{23}$. A nivel regional se evidenció caída del flujo en la mayor parte de las regiones encefálicas, pero esta caída fue muy ostensible en las regiones talámicas. Ambos efectos de la droga sobre el FSC global y regional ya se produjeron con dosis bajas de la droga, no lográndose un mayor efecto al aumentar la dosis. Esta acción bien establecida de la dexmedetomidina en humanos sanos generaría indirectamente un efecto reductor de la PIC que podría ser beneficiosa en los pacientes con lesión neurológica y baja "compliance" cerebral.

Los únicos datos que contamos del uso de dexmedetomidina en pacientes con lesión neurológica han sido aportados por el trabajo de Talke y col ${ }^{27}$. Ellos no encontraron cambios significativos en la presión del líquido cefalorraquídeo a nivel lumbar en un grupo de pacientes con cirugía transesfenoidal de hipófisis. Aunque fue un estudio pionero en la investigación clínica de la droga resultó al mismo tiempo limitado por: 1) la población de pacientes elegida, seguramente con "compliance" intracraneal normal y 2) por la medida indirecta de la PIC a través de un catéter lumbar.

Nuestros datos, a pesar de lo reducido de la población estudiada, muestran escaso efecto de la droga sobre la hemodinámica intracraneal en una población de pacientes traumatizados sin hipertensión intracraneana en el momento de la inclusión del estudio. A diferencia del estudio de Talke $\mathrm{y} \mathrm{col}^{27}$, la población elegida está constituída por 12 pacientes en el $4^{\circ}$ día promedio de evolución de su TECG, por lo que puede suponerse un compromiso de la "compliance" intracraneal ${ }^{21}$ a pesar de una PIC inicial $<$ de $20 \mathrm{~mm}$ de Hg. El estudio así diseñado lo creemos adecuado ya que la dexmedetomidina no se postula como recurso específico para reducir la HIC. En cambio, estaría indicada como droga de "salida" en el despertar de estos pacientes, el cual puede presentarse muy alterado con excitación psicomotriz y a veces delirio ${ }^{6}$. Sus efectos de simpaticolisis, promoción de sedación consciente, su ausencia de depresión respiratoria así como su débil efecto amnésico la hacen ideal para el seguimiento de estos pacientes en esta etapa evolutiva. Sin embargo, y a priori, no se podía descartar una reacción nociva de la droga sobre la hemodinámica intracraneal no totalmente normalizada aún, en esta etapa evolutiva de un TECG ${ }^{21}$.

El estudio mostró una tendencia a la disminución de la PPC sin alteración estadísticamente significativa de la PIC y PAM durante la infusión de dexmedetomidina a las dosis estudiadas. Esta acción marginal de la droga podría explicarse: por la suma de efectos no significativos individualmente sobre la PIC y PAM, o por la limitación metodológica vinculada al pequeño número de pacientes estudiados (error de tipo II). Sin embargo, cabe enfatizar que los valores de PPC nunca fueron menores a $60 \mathrm{mmHg}$, lo cual constituye un objetivo terapéutico fundamental en este tipo de pacientes. Este débil efecto sobre la PPC no condicionó alteraciones en el balance global de $\mathrm{O}_{2}$ estimado globalmente mediante los valores de $\mathrm{SjO}_{2}$ y $\mathrm{CEO}_{2}$ que variaron poco, indicando además, probablemente una conservación de los mecanismos autorregulatorios bajo la droga a las dosis utilizadas.

La FC disminuyó en forma significativa con respecto a los valores basales, probablemente debido al efecto simpaticolítico de la dexmedetomidina y sumado probablemente a efectos parasimpáticos directos de la droga actuando sobre los receptores $\alpha 2$ en los núcleos dorsal del vago y del tracto solitario ${ }^{22}$.

Nuestros hallazgos no pueden ser extrapolados a dosis mayores de las estipuladas por el laboratorio fabricante que han sido reportadas. $(1,2-2,5 \mathrm{ug} / \mathrm{kg} / \mathrm{h})^{12,28}$.

Aunque no ha sido demostrada aún la utilidad de la dexmedetomidina en comparación a los planes clásicos en el manejo del despertar de los enfermos neurocríticos ${ }^{26}$, nuestro estudio señala que la droga podría usarse sin mayores riesgos en este contexto.

Como conclusión, a las dosis utilizadas, la dexmedetomidina no se asocia con alteraciones significativas de la hemodinámica intracraneal ni del metabolismo global de oxígeno cerebral, a pesar de que el estudio se realizó en la etapa aguda del TECG, donde se presumen alteraciones de la "compliance" intracraneal y alteraciones de la reactividad vascular encefálica.

\section{Bibliografía}

1. Bachand, R., Scholz, J., Pinaud, M., et al.: The effects of dexmedetomidine in patientes in the intensive care setting. Intensive Care Med. 1999; 25 Suppl. 1: S 160.

2. Belleville, J.P., Ward, D.S., Bloor, B.C., et al.: Effects of 
intravenous dexmedetomidine in humans. I. Sedation, ventilation, and metabolic rate. Anesthesiology $1992 ; 77$ : 1125-1133.

3. Bhana, N., Goa, K., Mc Clellan, K.: Dexmedetomidine. Drugs. $2000 ; 59: 263-268$.

4. Bloor, B.C., Ward, D.S., Belleville, J.P., et al.: Effects of intravenous dexmedetomidine in humans. II. Hemodynamic changes. Anesthesiology 1992; 77: 1134-1142.

5. Coursin, D.B., Coursin, D.B., Maccioli, G.A.: Dexmedetomidine. Curr Opin Crit Care. 2001; 7: 221-226.

6. Ely, E.W., Siegel, M.D., Inouye, S.K.: Delirium in the intensive care unit: An under-recognized syndrome of organ dysfunction. Seminars in Respiratory \& Critical Care Medicine. 2001; 22:115-126.

7. Engelhard, K., Werner, C., Kaspar, S., Mollenberg, O., Blobner, M., Bachl, M., Kochs, E.: Effect of the alpha2-agonist dexmedetomidine on cerebral neurotransmitter concentrations during cerebral ischemia in rats. Anesthesiology. 2002; 96: 450-457.

8. Engelhard, K., Werner, C., Eberspacher, E., Bachl, M., Hildt, E., et al. Blobner, M.: The effect of the alpha 2-agonist dexmedetomidine and the N-methyl-D-aspartate antagonist $\mathrm{S}(+)$-ketamine on the expression of apoptosis-regulating proteins after incomplete cerebral ischemia and reperfusion in rats. Anesth Analg. 2003; 96: 524-531.

9. Ganjoo, P., Farber, N., Hudetz, A., Smith, J.J., Samso, E., Kampine, J. et al.: In Vivo Effects of Dexmedetomidine on Laser-Doppler Flow and Pial Arteriolar Diameter. Anesthesiology. 1998; 88: 429-439.

10. Iida, H., Iida, M., Ohata, H., Nagase, K., Dohi, S.: Hypothermia attenuates tha vasodilator effects of Dexmetomidine on pial vessels in rabbits in vivo. Anesth Analg 2004; 98: 477-482.

11. Jolkkonen, J., Puurunen, K., Koistinaho, J., Kauppinen, R., Haapalinna, A., Nieminen, L., et al.: Neuroprotection by the alpha2-adrenoceptor agonist, dexmedetomidine, in rat focal cerebral ischemia. Eur J Pharmacol. 1999 7; 372: 31-36.

12. Jorden, V.S.B., Tung, A.: Dexmedetomidine : Clinical Update Anesth Periop Mesd and Pain 2002; 21: 265-274.

13. Kamibayashi, T., Maze, M.: Clinical uses of $\alpha 2$ adrenergic agonists. Anesthesiology 2000; 93: 1345-1349.

14. Katsanoulas, C.: Redefining intensive care unit sedation. Internacional Congress and Symposium Series - Redefining sedation. 1998; 221: 83-88.

15. Kelly, D.F., Goodale, D.B., Williams, J., Herr, D.L., Chappel, E.T., Rosner, M.J., et al.: Propofol in the treatment of moderate and severe head injury: a randomized, prospective double-blinded pilot trial. J Neurosurg. 1999; 90: 1042-1052.

16. Knaus, D.A., Draper, E.A., Wagner, D.P., Zimmerman, J.E. APACHE II: A severity of disease classification system. Crit Care Med. 1985; 13: 818-829

17. Kuhmonen, J., Pokorny, J., Miettinen, R., Haapalinna, A., Jolkkonen, J., Riekkinen, P. Sr et al.: Neuroprotective effects of Dexmedetomidine in the Gerbil hippocampus after transient global ischemia. Anesthesiology 1997; 87: 371-377.
18. Louvelle, J.M.: Sedation in the intensive care unit: an overview. Can J Hosp Pharm. 1995; 48: 344-347.

19. Maier, C., Steinberg, G.K., Sun, G.H., Zhi, G.T., Maze, M.: Neuroprotection by the alpha 2-adrenoreceptor agonist dexmedetomidine in a focal model of cerebral ischemia. Anesthesiology. 1993; 79: 306-312.

20. Marshall, L.F., Marshall, S.B., Klauber, M.R., van Berkum Clark, M.: A new classification of head injury based on computerized tomography. J Neurosurg 1991; 75 (Suppl): S14-S20.

21. Maset, A.L., Maramarou, A., Ward, J.D., Choi, S., Lutz, H.A., Brooks, D,: Pressure-volume index inhead injury patients. J Neurosurg 1987; 67: 832-840.

22. Pentittila, J., Helminen, A., Anttila, M., Hinkka, S., Scheinin, H.: Cardiovascular and parasympathetic effects of dexmedetomidina in healthy subjects. Can J Physiol Pharmacol 2004 ; 82: 359-362

23. Prielipp, R.C., Wall, M.H., Tobin, J.R., Groban, L., Cannon, M.A., Fahey, F.H., et al.: Dexmedetomidine-induced sedation in volunteers decreases regional and global cerebral blood flow. Anesth Analg. 2002; 95: 1052-1059.

24. Sahuquillo, J., Biestro, A., Mena, M.P., Amoros, S., Lung, M., Poca, M.A. et al.: First tier measures in the treatment of intracranial hypertension in the patient with severe craniocerebral trauma. Proposal and justification of a protocol. Neurocirugia. 2002; 13: 78-100.

25. Shapiro, B.A., Warren, J., Egol, A.B., et al.: Practice parameters for intravenous analgesia and sedation for adult patients in the intensive care unit: an executive summary. Crit Care Med. 1995; 23: 1596-600.

26. Shelly, M.P.: Dexmedetomidine: a real innovation or more of the same? Editorial Br J Anaesth 2001; 87: 677-678.

27. Talke, P., Tong, C., Lee, H.W., Caldwell, J., Eisenach, J.C., Richardson, C.A.: Effect of Dexmedetomidine on lumbar cerebrospinal fluid pressure in humans. Anesth Analg. 1997; 85: 358-364.

28. Venn, R.M., Newman, P.J., Grounds, R.M.: A phase II study to evaluate the efficacy of Dexmedetomidine for sedation in the medical intensive care unit. Int Care Med 2003; 29: 201-207.

29. Wheeler, A.P.: Sedation, analgesia and paralysis in the intensive care unit. Chest. 1993; 104: 566-577.

30. Wijeysundera, D.N., Naik, J.S., Scott Beattie, W.: Alpha-2 adrenergic agonist to prevent perioperative cardiovascular complications: A Meta-analysis. Am J Med 2003; 114: 742-752.

Grille, P.; Biestro, A.; Fariña, G.; Miraballes, R.: Efectos de la dexmedetomidina sobre la hemodinámica intracraneal en pacientes con lesión encefálica traumatica grave. Neurocirugía 2005; 16: 411-418.

Correspondencia postal: Dr. Alberto Biestro. CTI del Hospital de Clínicas, Piso 14. Universidad de la República. Avenida Italia s/n. CP 11600 Montevideo. Uruguay. 
Comentario al trabajo: Efectos de la dexmedetomidina sobre la hemodinamia intracraneana en pacientes con lesión encefálica traumática grave de Grille y cols.

Al igual que otras muchas recomendaciones terapéuticas relacionadas con el traumatismo craneoencefálico grave (TCE), la sedación, habitualmente asociada a la analgesia, a pesar de no reunir un grado de evidencia suficiente para conseguir el nivel de estándar, forma parte de las guías clínicas como tratamiento no cuestionado. Diversas razones fundamentan dicha práctica en el neurointensivismo en general y en el TCE en particular. Existen indicaciones generales para el uso de los sedantes, idénticas a cualquier paciente crítico, como facilitación de la ventilación mecánica y de otras medidas terapéuticas invasivas, control de la ansiedad y agitación, consecución de cierto grado de amnesia, etc., e indicaciones específicas, debidas a los efectos neurofisiológicos de los sedantes, como: coadyuvante al tratamiento de la hipertensión intracraneal, descenso de la excitabilidad cortical, disminución de los requerimientos metabólicos cerebrales ${ }^{2}$, todo lo cual conlleva descenso de los insultos cerebrales secundarios y beneficio para la recuperación del tejido cerebral. Sin embargo, los particulares efectos que se exigen a la sedación en el TCE, expuestas en el presente número de Neurocirugía por Grille et al. ${ }^{5}$ como, entre otras, efecto de corta duración que produzca "ventana terapéutica" para el examen clínico; no alteración de la dinámica y hemodinámica intracraneal; simpaticolisis; y control de la excitación psicomotriz en la fase de retirada de la sedación, -tan frecuentemente observada cuando se emplean las benzodiacepinas-, justifica el debate en torno a la sedación y la búsqueda del agente ideal en el TCE.

El tipo de sedante, el opiáceo más adecuado al que asociarse, la duración y forma de interrupción de la sedación $\mathrm{y}$, finalmente, tras la aparición de remifentanilo, si podría cambiarse el régimen tradicional (hipnóticos + opiáceos) a sedación basada en la analgesia exclusivamente, son los aspectos más discutidos ${ }^{3}$. Para dar respuesta a las cuestiones planteadas son escasos los estudios de sedación en neurointensivismo, en contraposición al gran número de estudios realizados en otros grupos de pacientes críticos. Por ello el trabajo del grupo uruguayo nos parece interesante por el tema y el fármaco ensayado.

La dexmedetomidina, un agonista de los adrenorreceptores $\alpha 2$ con efectos analgésicos y sedantes, con un buen perfil farmacocinético y propiedades neuroprotectoras en animales de experimentación ${ }^{4}$, se emplea desde hace varios años en anestesia y cuidados intensivos, de forma habitual, cuando se requiere sedoanalgesia en cortos periodos de tiempo. El trabajo que comentamos, a pesar del escaso tamaño de la muestra, nos abre la posibilidad, sin riesgos de interferencia negativa en la PIC u otros parámetros hemometabólicos, del empleo de dexmedetomidina en el periodo de retirada de la sedoanalgesia convencional, donde, como bien señalan los autores, se producen situaciones de agitación, delirio, descargas simpáticas, etc., que obligan a retrogradar en la evolución prevista del paciente, con alargamiento de estancia en $\mathrm{UCl}$ y riesgo de nuevas complicaciones. Sin embargo, persisten interrogantes que los autores no disipan con sus resultados. ¿Cuánto tiempo podríamos mantener con seguridad la infusión? En caso de elevarse la PIC, ¿Qué actitud seguir? ¿Qué efectos podrían esperarse si precisamos su asociación a opiáceos? Y así algunos interrogantes más. No obstante, la experiencia del grupo de Lund ${ }^{1}$ con la clonidina, un agente, asimismo, $\alpha 2$ agonista de la que se resaltan sus propiedades coadyuvantes en el control de la PIC, justificarían nuevos estudios con dexmedetomidina, con mayor número de pacientes, durante periodos de tiempo más prolongado y en las condiciones habituales del paciente con TCE grave.

\section{Bibliografía}

1. Asgeirsson, B., Grände, P.O., Nordström, C.H.: A new therapy of post-trauma brain oedema based on haemodynamic principies for brain volume regulation. Intensive Care Med 1994; 20: 260-267.

2. Chiolero, R.L., Schoettker, P.: Sedation interruption in neurointensive care. Ann Fr Anesth Reanim 2004; 23: 541545.

3. Grille, P., Biestro, A., Fariña, G., Miraballes, R.: Efectos de la dexmedetomidina sobre la hemodinamia intracraneana en pacientes con lesión encefálica traumática grave. Neurocirugía 2005; 16:

4. Laudenbach, V., Mantz, J., Lagercrantz, H., Desmonts, J.M., Evrard, P., Gressens, P.:Effects of alpha (2)-adrenoreceptor agonists on perinatal excitotoxic brain injury: comparison of clonidine and dexmedetomidine. Anesthesiology 2002; 96 : 134-141.

5. Típps, L.B., Coplin, W.M., Murry, K.R., Rhoney, D.H.: Safety and feasibility of continuous infusion of remifentanil in the neurosurgical intensive care unit. Neurosurgery 2000; 46: 596-601. 